\title{
Swing Kinematics in Skilled Male Golfers Following Putting Practice
}

$\mathbf{T}$ o maintain a high level of skill, professional and elite golfers also spend considerable time playing and practicing, hitting up to 300 balls during a single practice session, ${ }^{29}$ and up to 2000 balls per week. ${ }^{12}$ Golfers spend considerable time practicing putting. ${ }^{17}$ Compared to shots with other clubs, the putting stroke involves the most sustained, as well as the greatest magnitude of, trunk inclination and forward flexion, but the least amount of trunk

motion. ${ }^{6}$ While trunk muscle activity during putting has not been specifically

STUDY DESIGN: Control laboratory study consisting of preintervention and postintervention measurements.

OBJECTIVE: To determine the effects of a putting practice session on the kinematics of full golf swings made by skilled male golfers.

BACKGROUND: Skilled golfers perform putting practice for prolonged periods. The combination of sustained trunk flexion with minimal trunk motion may affect the endurance capacity of the trunk extensor muscles. Because of their important role in the golf swing, any impairment of the trunk extensors may negatively influence full-swing kinematics, but this has not been previously evaluated.

METHODS AND MEASURES: Three-dimensional swing kinematics and holding time on the Biering-S $\phi$ rensen test of isometric trunk extensor endurance were evaluated in 29 skilled male golfers before and after performing a 40-minute putting task.

- RESULTS: After the intervention, peak segmental speeds were reduced and total swing duration increased (mean $\pm S D, 36 \pm 55$ milliseconds). examined, it has been shown that activity in the trunk extensors increases as

There were reductions in the magnitude of pelvis and torso axial rotation during the downswing (mean $\pm \mathrm{SD},-2.3^{\circ} \pm 2.6^{\circ}$ and $-2.3^{\circ} \pm 4.7^{\circ}$, respectively). The peak difference between torso rotation and pelvis rotation during early downswing was also significantly reduced by $0.9^{\circ} \pm 2.0^{\circ}$ $(P<.05)$. The effects on pelvis and torso rotation were smallest for golfers with higher body mass index (BMI). Holding time on the Biering-S $\phi$ rensen test after putting practice was reduced by $25.7 \pm$ 23.8 seconds $(P=.01)$.

CONCLUSION: Changes in swing kinematics observed following 40 minutes of putting practice might have resulted from fatigue-related impairment of the trunk extensor muscles, a view supported by the poorer performance on the postintervention Biering-Sфrensen test. Results showed that swing kinematics of golfers with high $\mathrm{BMI}$ were least affected by the putting practice.

- LEVEL OF EVIDENCE: Harm, level 5. J Orthop Sports Phys Ther 2008;38(7):425-433. doi:10.2519/jospt.2008.2617

KEY WORDS: golf, erector spinae, lumbar spine the trunk flexes forward from upright standing. ${ }^{14,28}$ Putting, therefore, is likely to involve significant trunk extensor activity, and performing putting practice for prolonged periods may result in fatigue of these muscles. The trunk and hip extensors, specifically the erector spinae, hamstrings, and gluteal muscles, all play a significant role in producing and controlling trunk and pelvis motion during the full-swing action. So fatigue of these muscles, as is hypothesized to occur following putting practice, may affect the kinematics of the full golf swing.,18,22,31

Relationships between golf performance and the range, sequencing, and timing of rotation of the torso and the pelvis during the swing have been widely studied, particularly as these factors are thought to be important in the generation of power in the golf swing. ${ }^{3,4,19,21,24}$ For example, the magnitude of the difference between torso and pelvis rotation increases during the backswing but among elite players it typically peaks in the early part of the downswing, as the pelvis begins rotating towards the target before the torso. ${ }^{3,19}$ The magnitude of the torso-pelvis differential, as well as the rate at which it occurs during the backswing, and the rate at which it is reduced during the downswing, are thought to be important variables for obtaining longer driving distances, possibly achieved through

\footnotetext{
${ }^{1}$ Lecturer, School of Physiotherapy and Exercise Science, Griffith University, Gold Coast, Australia. ${ }^{2}$ Professor, Acting Director, Research and Innovation and Associate Dean, The University of Sydney, Sydney, Australia. ${ }^{3}$ Senior Lecturer, The University of Sydney, Sydney, Australia. ${ }^{4}$ Associate Professor, School of Physiotherapy and Exercise Science, Griffith University, Gold Coast, Australia. We affirm that we have no financial affiliation or involvement with any commercial organization that has a direct financial interest in any matter included in this manuscript, nor do we have any other conflict of interest (such as personal associations or involvement as a director, officer or expert witness). This study was approved by the Griffith University Human Research Ethics Committee. Address correspondence to Kerrie Evans, School of Physiotherapy and Exercise Science, Griffith University, Gold Coast Campus, PMB 50, Gold Coast Mail Centre QLD, 9726, Australia. E-mail: Kerrie.Evans@griffith.edu.au
} 
more effective use of stretch-shorten cycle (SSC) contractions. ${ }^{3,4}$ That is, rapidly stretching the muscles of both the trunk and the pelvis during the backswing, followed immediately by concentric contractions of these same muscles, may enhance power production during the downswing. ${ }^{11,30}$ The trunk extensors, one of the important muscle groups involved in the golf swing, demonstrate significant activity both during the backswing and downswing and may play a critical role in the effective use of the SSC. ${ }^{1,11,18,22,31}$ Thus the effects of prolonged activation of these muscles, as is hypothesized to occur during sustained putting, may affect the ability of the golfer to utilize the SSC.

A proximal-distal sequence of body segment rotations, whereby the pelvis reaches peak angular velocity first, followed by the torso and finally the wrists, has been shown to maximize club head speed at impact. ${ }^{3,21,27}$ Less is known regarding the ideal duration of delays between peak segmental speeds. However, given that previous research has suggested that muscle fatigue affects the temporal characteristics of other multijoint sports tasks, ${ }^{8}$ it may be that it is in the precision of timing and sequencing between the trunk and the pelvis where the effects of fatigue become apparent.

The purpose of this study was to determine the effect of 40 minutes of putting practice on swing kinematics in skilled male golfers. We anticipated that the putting task would affect pelvis and torso kinematics, and decrease hand speed during the swing. We also anticipated that the effects on performance would be a result of fatigue of the trunk extensors and would therefore result in poorer performance on a subsequent Biering-Sørensen test. ${ }^{2}$ The Biering-Sørensen test is an isometric trunk extensor endurance test frequently used to examine the fatigability of the trunk extensors in clinical environments. ${ }^{5,15}$ This test has been shown to have good reliability and high correlation between holding time on the test and electromyographic (EMG) fatigue parameters, such as median frequency decline. ${ }^{13-16,20}$ Lastly, the golfer's body mass index (BMI) was considered. While the influence of player anthropometrics on swing kinematics has not previously been widely investigated, an association between being a golfer with low BMI and experiencing low back pain (LBP) has been reported. ${ }^{7,17}$ Alternatively, it has been hypothesized that during trunk flexion, a higher BMI increases the rotational moment around the lumbosacral joint, requiring greater activity in the erector spinae muscles. ${ }^{14}$ Accordingly, a second aim of the present study was to determine whether the golfer's BMI influenced any changes observed in swing kinematics following the putting practice.

\section{METHODS}

\section{Participants}

M ALE GOLFERS ( $\mathrm{N}=29$ ) WITH A mean \pm SD Australian handicap of $1.6 \pm 2.4$ (range, $0-8$ ) strokes volunteered (TABLE 1). At this level of skill, the kinematics of repeated swings was expected to be consistent. ${ }^{10,23,26}$ Nineteen of the participants were qualified golf professionals or trainee professionals, credited with a handicap of 0 , and 10 participants had handicaps equal to or less than 8 . All participants were right-handed, with BMI values between 20.5 and $31.7 \mathrm{~kg} / \mathrm{m}^{2}$. Twenty-five of the participants reported having experienced previous episodes of LBP, but all were symptom free at the time of testing. Written consent was obtained prior to data collection and approval to conduct the study was given by The Griffith University Human Research Ethics Committee.

\section{Experimental Design}

All participants performed the same warm-up routine, consisting of $10 \mathrm{~min}$ utes of stretching the muscles of the torso and upper and lower limbs, followed by 5 practice swings, using a 5 -iron to optimize swing consistency. Participants were then told to imagine that they had hit their first shot down the middle of the fairway and had chosen a 5-iron to make their next shot. They were asked to hit the ball as straight as possible. Kinematic data for the pelvis, torso, head, and hand were then collected from 5 full swings, with participants using their own club, hitting golf balls off a mat into a net. Participants paused for 30 seconds between each swing.

Following this, participants performed the Biering-Sørensen test and a 40-minute putting task. The duration of the putting task based on practice times of current participants (TABLE 1) and previously reported data. ${ }^{17}$ The putting task was self-paced and involved hitting balls along a mat to a hole $3 \mathrm{~m}$ away. Immediately after the putting session, the participants performed another 5 full swings with the same golf club, again with the intent to hit each ball as straight as possible. Lastly, the Biering-Sørensen test was repeated to determine any changes in trunk extensor endurance.

\section{Data Collection Procedures}

Biering-Sørensen Test Participants were positioned prone with the anterior superior iliac spines resting on the edge of a plinth and the upper body resting on a chair. The lower body was fixed to the plinth by 3 seat belts. Participants crossed the arms over the chest, lifted the upper body to horizontal, and maintained this position for as long as possible. They were encouraged throughout the test and given feedback if they deviated from horizontal. The test was terminated if the position could not be maintained due to fatigue or excessive discomfort. Holding time was recorded in seconds.

Kinematic Data Golf swing 3-dimensional (3-D) kinematic data were sampled at $240 \mathrm{~Hz}$ using the Polhemus Liberty electromagnetic tracking system (Polhemus Inc, Colchester, VT), with Skill-3D for Golf software (Skill Technologies Inc, Phoenix, AZ). The Polhemus system has a manufacturer-specified static accuracy of $0.76 \mathrm{~mm}$ along each sensor axis and $0.15^{\circ}$ root-mean-square (RMS) for sensor orientation, with a stated resolution of 0.04 $\mathrm{mm}$ for position and $0.0012^{\circ}$ for orienta- 


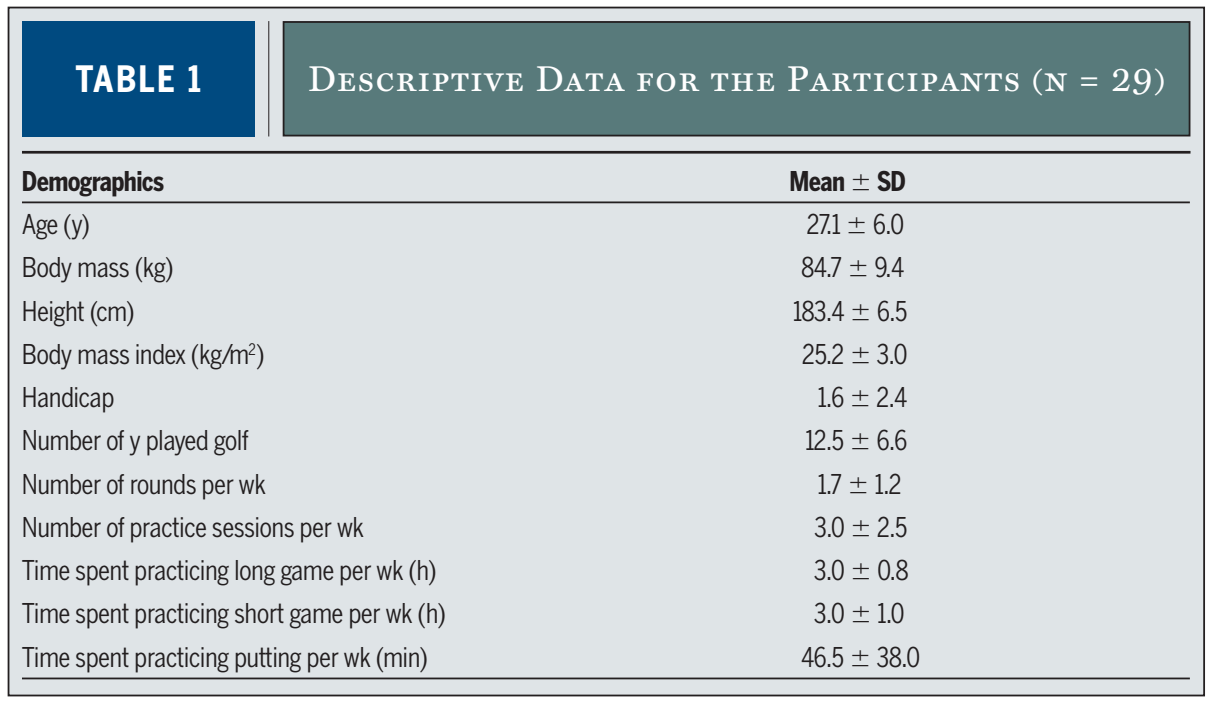

tion. The origin of the global coordinate system (transmitter) was approximately $0.3 \mathrm{~m}$ behind the golfer, with $+z$ vertically upwards, $+y$ the target direction, and $+x$ directed anteriorly. Four 6-degrees-offreedom $(6 \mathrm{DOF})$ sensors were used to record the 3 -D linear $(x, y, z)$ and angular (pitch, yaw, roll) motion of the pelvis, torso, head, and left hand. To represent body segments, sensors were placed on the forehead $1 \mathrm{~cm}$ above the bridge of the nose (head), the thoracic spine at the level of T3 (torso), the sacrum at S2 (pelvis), and the posterior aspect of the left hand (hand). So as not to impede the swing, sensors were secured using a Velcro strap around the forehead for the forehead sensor, a harness for the sensors at T3 and $\mathrm{S} 2$, and the participant's golf glove for the hand sensor (FIGURE 1). Prior to collecting the kinematic data, calibration trials were recorded with participants in an upright, neutral standing position facing the $+x$ axis. The harness and sensors were removed following the preintervention trials and then reapplied for the postintervention assessment.

\section{Data Analysis Procedures}

All kinematic analysis was performed using custom-designed software (Golf BioDynamics Pty Ltd, Corinda, Australia) developed with Matlab, Version 7.0.4 (The Mathworks, Inc, Natwick, MA). In the present study, the start of the swing was defined from movement of the hand sensor in the $-y$ direction - that is, movement away from target, and the top of swing by maximal pelvis rotation from target. Impact was defined as the lowest vertical point of the hand sensor in the $z$ direction. The backswing and downswing were defined as the time between the start of backswing and the top of the swing and from top of swing to impact, respectively.

Rotations All orientations were expressed relative to the upright, neutral standing position. This was achieved by performing a transformation to align the coordinate system for each sensor with the global coordinate system (GCS) in the neutral position. Head, torso, and pelvis axial rotations were subsequently obtained from projections of their respective $x$-axis unit vectors onto the $x$ - $y$ plane of the GCS, with rotations towards target (counterclockwise rotation) defined as positive. The maximum difference between the magnitude of torso rotation and pelvis rotation was calculated (the "X-Factor") and the amount by which this value increased from the top of the backswing to the maximum differential between torso and pelvis rotation during the early part of the downswing (the "X-Factor stretch") was also calculated.,19 The maximum rate at which the X-Factor stretch was generated and the rate at which this difference decreased during

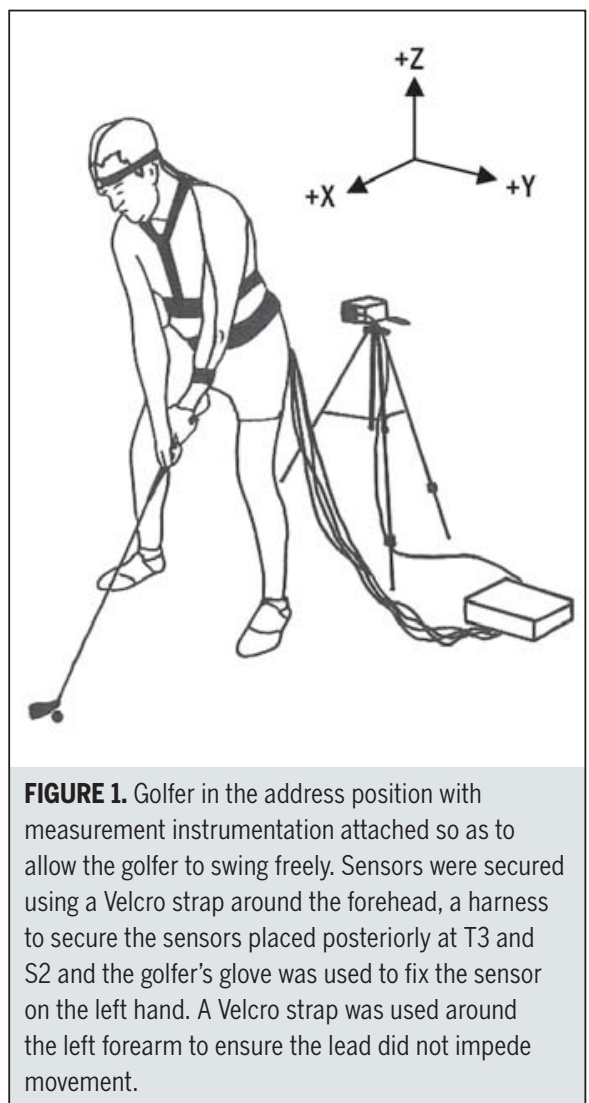

the downswing ("rate of recoil") were calculated from the slope of the X-Factor curve. Head, torso, and pelvis flexion and lateral flexion were obtained from the angle between the respective horizontal sensor axes and the global $x-y$ plane. Positive values for flexion and lateral flexion angles indicated forward flexion and lateral flexion to the right, respectively.

Displacements Displacements of the head, torso, and pelvis along the $x$ (anterior/posterior), $y$ (left/right), and $z$ (up/ down) axes of the GCS were expressed relative to the address position, the position a golfer adopts prior to initiating a swing.

Speed and Relative Timing Hand speed in $\mathrm{m} \cdot \mathrm{s}^{-1}$ was obtained from the rate of change of the resultant displacement of the hand sensor. Pelvis and torso speed were calculated in $\mathrm{deg} \cdot \mathrm{s}^{-1}$ from the slope of the corresponding angle-time curves. Lag times in milliseconds were computed for the time between the peak linear velocity of the hand and impact (hand lag time), the peak angular velocity of the torso and peak linear velocity of the 


\begin{tabular}{|c|c|c|c|c|c|}
\hline \multirow[t]{2}{*}{ TABLE 2} & \multicolumn{5}{|c|}{$\begin{array}{c}\text { VALUES FOR THE VARIABLES MEASURED } \\
\text { DURING THE BACKSWING* }\end{array}$} \\
\hline & Preintervention & Postintervention & P Value $^{\dagger}$ & Difference $^{\ddagger}$ & $95 \% \mathrm{Cl}$ \\
\hline \multicolumn{6}{|l|}{$\overline{R o t a t i o n s ~(d e g) ~}$} \\
\hline Head rotation & $-19.0 \pm 11.4$ & $-20.3 \pm 12.4$ & .11 & $-1.3 \pm 4.1$ & -2.8 to 0.3 \\
\hline Torso rotation & $-86.5 \pm 7.2$ & $-84.5 \pm 7.9$ & $.01^{\S}$ & $2.0 \pm 3.6$ & 0.5 to 3.3 \\
\hline Torso flexion & $7.7 \pm 9.2$ & $7.9 \pm 10.0$ & .81 & $0.2 \pm 3.6$ & -1.2 to 1.5 \\
\hline Pelvis rotation & $-45.4 \pm 9.3$ & $-44.4 \pm 8.8$ & .15 & $1.0 \pm 3.6$ & -0.4 to 2.3 \\
\hline Pelvis flexion & $16.0 \pm 6.8$ & $14.8 \pm 6.3$ & .17 & $-1.2 \pm 4.7$ & -3.0 to 0.6 \\
\hline X-Factor & $-41.1 \pm 6.7$ & $-40.1 \pm 6.3$ & .31 & $1.0 \pm 4.9$ & -0.9 to 2.8 \\
\hline \multicolumn{6}{|l|}{ Displacements (cm) } \\
\hline Head anterior/posterior & $r \quad 1.3 \pm 2.6$ & $1.3 \pm 2.6$ & .93 & $0.0 \pm 0.6$ & -0.3 to 0.2 \\
\hline Head left/right & $6.8 \pm 4.4$ & $6.9 \pm 4.5$ & .60 & $0.1 \pm 0.9$ & -0.3 to 0.5 \\
\hline Head up/down & $2.0 \pm 3.9$ & $1.8 \pm 4.0$ & $.04 \S$ & $-0.2 \pm 0.6$ & -0.5 to 0.0 \\
\hline Torso left/right & $-8.0 \pm 3.6$ & $-8.2 \pm 3.7$ & .59 & $-0.2 \pm 1.6$ & -0.7 to 0.4 \\
\hline Pelvis anterior/posterior & r $\quad 5.4 \pm 5.2$ & $6.1 \pm 2.0$ & .43 & $0.7 \pm 4.8$ & -1.1 to 2.6 \\
\hline Pelvis left/right & $-5.8 \pm 3.1$ & $-6.2 \pm 3.2$ & .14 & $-0.4 \pm 1.3$ & -0.8 to 0.1 \\
\hline Pelvis up/down & $-2.6 \pm 1.8$ & $-3.1 \pm 3.3$ & .50 & $-0.5 \pm 4.8$ & -1.1 to 2.6 \\
\hline \multicolumn{6}{|c|}{$\begin{array}{l}\text { * All values, except those of } \mathrm{P} \text { value and } 95 \% \text { CI, are expressed as mean } \pm S D . \\
{ }^{+} \mathrm{P} \text { values relate to the } F \text { tests for the preintervention-postintervention differences. } \\
{ }^{*} \text { Postintervention minus preintervention. } \\
\S \text { Significant differences }(\mathrm{P}<.05) \text {. }\end{array}$} \\
\hline
\end{tabular}

\begin{tabular}{|c|c|c|c|c|c|}
\hline \multirow[t]{2}{*}{ TABLE 3} & \multicolumn{5}{|c|}{$\begin{array}{c}\text { VALUES FOR THE VARIABLES MEASURED } \\
\text { DURING THE DOWNSWING* }\end{array}$} \\
\hline & Preintervention & Postintervention & $P$ Value $^{\dagger}$ & Difference $^{\ddagger}$ & $95 \% \mathrm{Cl}$ \\
\hline \multicolumn{6}{|l|}{ Rotations } \\
\hline Head rotation (deg) & $9.6 \pm 12.2$ & $7.3 \pm 11.5$ & .09 & $-2.3 \pm 6.8$ & -4.9 to 0.3 \\
\hline Torso rotation (deg) & $20.4 \pm 8.3$ & $18.1 \pm 8.6$ & $.01 \S$ & $-2.3 \pm 4.7$ & -4.1 to -0.5 \\
\hline Torso flexion (deg) & $41.3 \pm 5.0$ & $39.1 \pm 9.0$ & .12 & $-2.2 \pm 7.5$ & -5.1 to 0.6 \\
\hline Pelvis rotation (deg) & $29.4 \pm 9.5$ & $27.1 \pm 8.9$ & $<.01^{\S}$ & $-2.3 \pm 2.6$ & -3.3 to -1.3 \\
\hline Pelvis lateral flexion (deg) & $10.2 \pm 2.7$ & $9.5 \pm 2.6$ & $.02^{\S}$ & $-0.7 \pm 1.5$ & -1.2 to -0.1 \\
\hline Pelvis flexion (deg) & $4.9 \pm 6.1$ & $4.9 \pm 5.4$ & .99 & $0.0 \pm 4.4$ & -1.7 to 1.7 \\
\hline X-Factor stretch (deg) & $-9.7 \pm 5.5$ & $-8.8 \pm 4.9$ & $.03 \S$ & $0.9 \pm 2.0$ & 0.1 to 1.7 \\
\hline Rate of recoil $\left(\mathrm{deg} \cdot \mathrm{s}^{-1}\right)$ & $393.2 \pm 74.8$ & $362.8 \pm 91.7$ & $<.01^{\S}$ & $-30.4 \pm 45.1$ & -47.5 to -13.2 \\
\hline Rate of stretch $\left(\mathrm{deg} \cdot \mathrm{s}^{-1}\right)$ & $-67.0 \pm 31.1$ & $-61.0 \pm 27.8$ & $.01^{\S}$ & $6.0 \pm 12.4$ & 1.4 to 10.8 \\
\hline \multicolumn{6}{|l|}{ Displacements } \\
\hline Head anterior/posterior (cm) & $-2.2 \pm 2.0$ & $-2.4 \pm 1.8$ & .18 & $-0.1 \pm 0.5$ & -0.3 to 0.1 \\
\hline Head up/down (cm) & $-3.5 \pm 3.1$ & $-3.1 \pm 3.3$ & .08 & $0.3 \pm 1.0$ & 0.0 to 0.7 \\
\hline Torso left/right (cm) & $-1.0 \pm 3.7$ & $-1.2 \pm 3.7$ & .43 & $-0.2 \pm 1.4$ & -0.7 to 0.3 \\
\hline Pelvis anterior/posterior $(\mathrm{cm})$ & $1.9 \pm 2.5$ & $1.6 \pm 2.8$ & .34 & $-0.3 \pm 1.8$ & -1.0 to 0.4 \\
\hline Pelvis left/right (cm) & $-2.4 \pm 3.7$ & $-3.1 \pm 3.7$ & $.01 \S$ & $-0.7 \pm 0.8$ & -1.0 to -0.3 \\
\hline \multicolumn{6}{|l|}{ Speed and relative timing } \\
\hline Hand speed $\left(\mathrm{m} \cdot \mathrm{s}^{-1}\right)$ & $5.56 \pm 0.43$ & $5.42 \pm 0.42$ & $<.01^{\S}$ & $-0.14 \pm 0.13$ & -0.19 to -0.10 \\
\hline Torso speed $\left(\mathrm{deg} \cdot \mathrm{s}^{-1}\right)$ & $426.0 \pm 38.4$ & $411.2 \pm 40.9$ & $<.01^{5}$ & $-14.8 \pm 12.0$ & -19.4 to -10.2 \\
\hline Pelvis speed (deg.s $\left.\mathrm{s}^{-1}\right)$ & $310.8 \pm 32.7$ & $295.4 \pm 33.5$ & $.01^{\S}$ & $-15.4 \pm 11.7$ & -19.8 to -10.9 \\
\hline Hand-lag time (ms) & $34.2 \pm 7.2$ & $35.6 \pm 7.4$ & $<.01^{\S}$ & $1.4 \pm 2.0$ & 0.6 to 2.1 \\
\hline Torso-hand lag time (ms) & $29.6 \pm 24.8$ & $27.7 \pm 28.9$ & .36 & $-1.9 \pm 11.4$ & -6.3 to 2.4 \\
\hline Pelvis-torso lag time (ms) & $31.6 \pm 26.5$ & $34.3 \pm 28.6$ & .35 & $2.7 \pm 15.2$ & -3.1 to 8.4 \\
\hline \multicolumn{6}{|c|}{$\begin{array}{l}\text { * All values, except those of } \mathrm{P} \text { value and } 95 \% \text { CI, are expressed as mean } \pm S D \text {. } \\
{ }^{+} P \text { values relate to the } F \text { tests for the preintervention-postintervention differences. } \\
{ }^{\ddagger} \text { Postintervention minus preintervention. } \\
{ }^{\S} \text { Significant differences }(\mathrm{P}<.05) \text {. }\end{array}$} \\
\hline
\end{tabular}

hand (torso-hand lag time), and the peak angular velocities of the pelvis and torso (pelvis-torso lag time).

\section{Statistical Analysis}

Means for each of the variables analyzed were calculated over the 5 swings performed before, and for the 5 swings performed after, the intervention. Eight variables were analyzed for the address position, 13 for the backswing (TABLE 2), 20 for the downswing (TABLE 3), and 3 for swing duration (TABLE 4). Intraclass correlation coefficient $\left(\mathrm{ICC}_{3,1}\right)$ reliability indices were computed both within the preswing and postswing trials. After entering the means of the 5 trials, a repeated-measures analysis of variance (ANOVA) was used to examine preintervention and postintervention differences in each of the defined variables. To explore the effect of BMI on the Biering-Sørensen test and swing kinematics, golfers were subdivided into $3 \mathrm{BMI}$ subgroups, by visual inspection of the histogram. The trend analysis option within SPSS ANOVA was selected where factor levels were on a continuum, as with BMI. SPSS Version 12.0.1 for Windows was used for all analyses and $P<.05$ was considered significant.

\section{RESULTS}

NTERTRIAL RELIABILITY VALUES OBtained for repeat measurements of variables associated with the swings preintervention ranged from 0.91 to 0.98 and, for the swings postintervention, from 0.87 to 0.98 .

\section{Swing Kinematics}

To depict the variables measured, and to illustrate the preintervention-postintervention differences, kinematic data for a golf swing performed before and after the intervention for a single participant are displayed in FIGURE 2. Several of the kinematic variables were significantly different between preintervention and postintervention.

Address Position In the address position, 


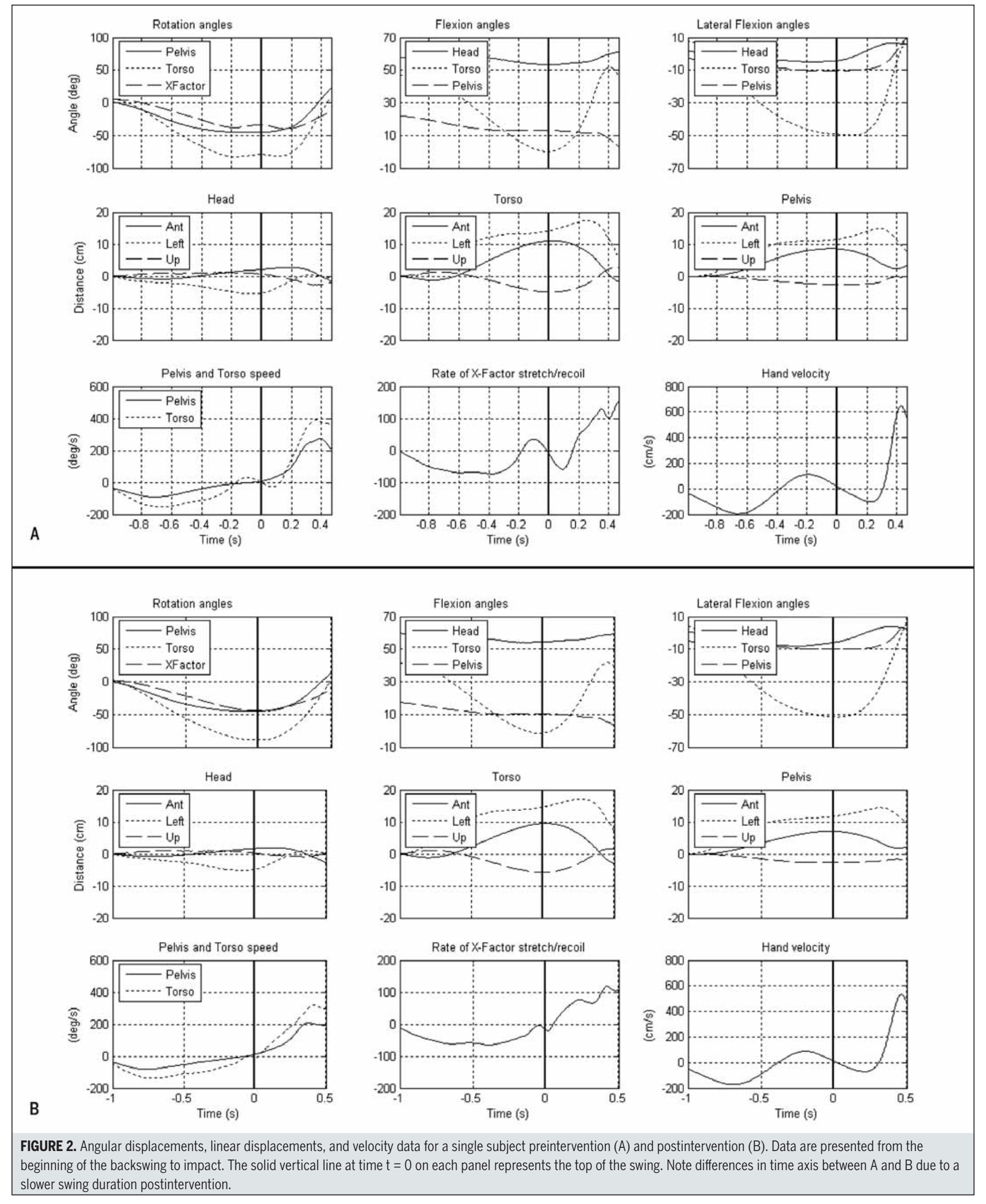




\section{[ RESEARCH REPORT ]}

\begin{tabular}{|c|c|c|c|c|c|}
\hline \multirow{2}{*}{ 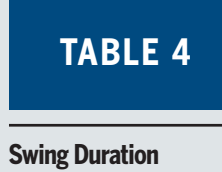 } & \multicolumn{5}{|c|}{$\begin{array}{c}\text { VAlues FOR Backswing, } \\
\text { Downswing, And Total Swing Time* }\end{array}$} \\
\hline & Preintervention & Postintervention & P Value $^{\dagger}$ & Difference ${ }^{\ddagger}$ & $95 \% \mathrm{Cl}$ \\
\hline Backswing (ms) & $1045 \pm 201$ & $1075 \pm 228$ & $<.01^{\S}$ & $30 \pm 52$ & 10.3 to 49.7 \\
\hline Downswing (ms) & $417 \pm 54$ & $423 \pm 53$ & .12 & $6 \pm 18$ & -1.2 to 12.2 \\
\hline Total swing time (ms) & $1462 \pm 226$ & $1498 \pm 242$ & $<.01^{\S}$ & $36 \pm 55$ & 14.6 to 56.2 \\
\hline \multicolumn{6}{|c|}{$\begin{array}{l}\text { * All values, except those of } \mathrm{P} \text { value and } 95 \% \text { CI, are expressed as mean } \pm S D \text {. } \\
{ }^{+} \mathrm{P} \text { values relate to the } F \text { tests for the preintervention-postintervention differences. } \\
{ }^{\ddagger} \text { Postintervention minus preintervention. } \\
{ }^{\S} \text { Significant differences }(\mathrm{P}<.05) \text {. }\end{array}$} \\
\hline
\end{tabular}

\begin{tabular}{|c|c|c|c|}
\hline \multirow[t]{2}{*}{ TABLE 5} & \multicolumn{3}{|c|}{$\begin{array}{c}\text { Values For Downswing Torso and Pelvis } \\
\text { Rotation During 5-Iron Swings For the } 3 \text { Body } \\
\text { Mass Index (BMI) Subgroups }\end{array}$} \\
\hline & BMI-Low* & BMI-Medium ${ }^{\dagger}$ & BMI-High $^{\ddagger}$ \\
\hline \multicolumn{4}{|l|}{ Torso rotation (deg) } \\
\hline Preintervention§ & $22.8 \pm 8.3$ & $19.5 \pm 7.0$ & $19.4 \pm 9.4$ \\
\hline Postintervention $\S$ & $20.6 \pm 5.8$ & $14.2 \pm 9.1$ & $19.3 \pm 9.3$ \\
\hline Difference" & $-2.2^{\natural}$ & $-5.3^{9}$ & -0.1 \\
\hline \multicolumn{4}{|l|}{ Pelvis rotation (deg) } \\
\hline Preintervention ${ }^{\S}$ & $36.0 \pm 5.2$ & $28.1 \pm 10.8$ & $25.9 \pm 8.9$ \\
\hline Postintervention§ & $32.3 \pm 5.6$ & $25.1 \pm 10.2$ & $25.2 \pm 8.9$ \\
\hline Difference" & $-3.7 \uparrow$ & -3.01 & -0.7 \\
\hline \multicolumn{4}{|c|}{$\begin{array}{l}\text { * Range, } 19.9-22.9 \mathrm{~kg} / \mathrm{m}^{2} ; n=8 . \\
{ }^{*} \text { Range, } 23.3-25.7 \mathrm{~kg} / \mathrm{m}^{2} ; n=9 . \\
{ }^{*} \text { Range, } 26.6-31.7 \mathrm{~kg} / \mathrm{m}^{2} ; n=12 . \\
\text { \$ Values are mean } \pm S D . \\
\text { "Calculated as postintervention minus preintervention values. } \\
\text { " The preintervention-postintervention difference for golfers with low and medium BMI is significantly } \\
\text { greater than for those with high BMI }(\mathrm{P}<.05) \text {. }\end{array}$} \\
\hline
\end{tabular}

the only variables that were significantly different between preputting and postputting session were pelvis flexion $(P=$ $.02)$ and head lateral flexion $(P=.046)$. The pelvis was significantly less flexed postintervention (mean $\pm \mathrm{SD}, 21.3^{\circ} \pm$ $\left.6.9^{\circ}\right)$, compared to preintervention $\left(23.1^{\circ}\right.$ $\pm 7.1^{\circ}$ ), and the head was significantly less laterally flexed to the right postintervention $\left(0.5^{\circ} \pm 4.7^{\circ}\right)$ compared to $1.4^{\circ} \pm$ $5.8^{\circ}$ preintervention.

Backswing During the backswing there was a significant reduction in torso rotation $(P=.01)$ and upward head movement $(P=.04)$ postintervention (TABLE 2).

Downswing During the downswing (TABLE 3), rotation of the pelvis $(P<.01)$ and torso $(P=.01)$ were significantly decreased postintervention, as was the amount of pelvis lateral flexion $(P=.02)$.
There were also significant reductions in the X-Factor stretch $(P=.03)$, the rate of stretch $(P=.01)$, and the rate of recoil $(P<.01)$. Torso speed $(P<.01)$, pelvis speed $(P=.01)$, hand speed $(P<.01)$, and hand-lag $(P<.01)$ were all significantly slower (TABLE 3). The reductions in segment speeds, and rate of stretch and rate of recoil, are shown in FIGURE 2.

Swing Duration The proportion of the total swing time attributed to the backswing and downswing was $71.5 \%$ and $28.5 \%$ preintervention and $71.8 \%$ and $28.2 \%$ postintervention (TABLE 4 ). The total swing time, from address to impact, was significantly slower $(P<.01)$ by $36 \pm$ 55 milliseconds postintervention. There was no difference in mean downswing time, but mean backswing time was significantly slower by $30 \pm 52$ milliseconds
$(P<.01)$.

Trunk Extensor Endurance, BMI, and Swing Kinematics

Holding time on the Biering-Sørensen test was significantly lower $(P=.01)$ at the end of the study protocol (mean \pm $\mathrm{SD}, 123.4 \pm 33.2$ seconds) than at the beginning (149.2 \pm 37.8 seconds). When golfers were divided into 3 BMI subgroups (TABLE 5), mean BMI values were $21.8(\mathrm{n}=8), 24.3(\mathrm{n}=9)$, and $28.3(\mathrm{n}$ $=12) \mathrm{kg} / \mathrm{m}^{2}$. Trend analysis conducted within ANOVA showed a significant linear decrease in Biering-Sørensen holding time as BMI increased across subgroups $\left(\mathrm{F}_{1,26}=18.75, P<.001\right)$. There was also a postintervention reduction in holding time $\left(\mathrm{F}_{1,26}=39.39, P<.001\right)$, but no interaction between these 2 effects $\left(\mathrm{F}_{1,26}=\right.$ 2.0, $P=.16)$. Thus, after the putting task, performance on the endurance test was worse by a mean 26 seconds, regardless of BMI subgroup.

The amount of preinterventionpostintervention difference in 2 kinematic measures, torso and pelvis rotation (TABLE 5), was found to be significantly different according to BMI subgroup $\left(\mathrm{F}_{1,26}=4.56, P\right.$ $=.02$ and $\mathrm{F}_{1,26}=3.88, P=.03$, respectively). That is, golfers with low and medium BMI had significantly larger reductions in range of torso and pelvis rotation during the swings made after the putting task, compared to those with high BMI, who were relatively less affected.

\section{DISCUSSION}

N THIS STUdy, GOLFERS EXHIBITED systematic changes in full-swing kinematics following a 40-minute putting task. The putting task was used because golfers practice their putting skills for long periods, and, when competing, often perform putting drills immediately prior to teeing off. Further, putting has also been shown to significantly contribute to spinal loading during a round, ${ }^{6}$ so it has sport-specific validity to incorporate as a task prior to an examination of swing performance. While an effective putting 
stroke does not require body segments to move through large ranges of motion, as occurs in the full-swing action, it was hypothesized that the combination of sustained trunk flexion with minimal body movement would induce some fatigue of the trunk extensor muscles, resulting in immediate changes in kinematics of full swings. Given that the Biering-Sørensen test can normally be repeated within a short period, with no deleterious effects on performance ${ }^{25}$ the significantly lower holding times observed postintervention support the interpretation that $40 \mathrm{~min}$ utes of putting induced some fatigue of the trunk extensors. This interpretation could be further clarified using EMG because the negative effect on swing kinematics may have been due to central fatigue rather than peripheral fatigue.

Following the 40-minute putting task, there was significantly less torso rotation achieved during the backswing, a factor contributing to the significant reduction in the maximum difference between torso and pelvis rotation in the early part of the downswing. It has been suggested that the magnitude of the difference between torso and pelvic rotation, and its correlation with club head velocity, may be related to the SSC.,11 That is, for the majority of elite players, left pelvic rotation initiates the downswing before the arms and torso complete the backswing, effectively increasing the torso/pelvis differential, hence, the stretch on the muscles of the trunk. ${ }^{3}$ Thus, less rotational difference between the torso and the pelvis during the downswing may limit the golfer's ability to effectively use the SSC to enhance power production, ultimately decreasing hitting distance. EMG studies have demonstrated the important role of the trunk extensors during the downswing. The hip extensors assist pelvic rotation and, as the torso begins the downswing, activity of the erector spinae and abdominal muscles increases. ${ }^{1,22,31}$ Alterations in the ability of the trunk extensors to produce or control trunk and pelvis rotation, such as is hypothesized to have occurred following putting practice, may compromise the high-level motor control needed for effective golf performance.

In a SSC, optimal performance results when the rate of stretch is high. ${ }^{11}$ In the present study, putting for 40 minutes significantly reduced the rate of stretch during the swing and affected the temporal characteristics of the swing. The duration of the backswing and total swing were significantly higher postintervention, although the relative proportion of the swing phases was not affected. During the downswing, the range of torso and pelvis rotation, and the rate of recoil (how quickly the torso-pelvis rotation difference decreased) were also significantly reduced. The overall effect was that, during the swing after putting practice, the golfers were taking longer to move through a lesser amount of range of motion.

Interestingly, the changes in the magnitude of pelvis and torso rotation were smallest for golfers with high BMI. Prior to the putting task, the range of torso and pelvis rotation was already lower for the individuals in the high-BMI group. An inference arising from this finding is that the swing kinematics of golfers with low and high BMI are different, and so their swings are differentially affected by 40 minutes of putting practice. The fact that the greatest postintervention adjustments were made by the leanest golfers suggests that the golfers in this group are the most vulnerable to the effects of fatigue, and this is consistent with previous findings that leaner golfers were the most susceptible to LBP. ${ }^{717}$ One possibility is that the additional body mass of golfers with higher BMI not only provides them with a buffer against the repeated spinal rotational stresses generated during the swing but that the inertia of the additional mass acting during the swing also mitigates the effects of fatigue. Extending this view, it may be that it is the additional effort made by golfers with low BMI to maintain their swing characteristics when fatigued that increases their vulnerability to LBP.

The swing of elite golfers is characterized by a pattern of motion that conforms to the summation of forces principle, ${ }^{3}$ with maximum club head speed resulting from a sequential proximal-distal pattern of motion. ${ }^{27}$ While the sequence of segmental motion was not affected following the intervention, peak velocity for all segments was reduced and the timing at which these peaks occurred was significantly altered. For example, peak pelvis velocity occurred earlier relative to impact, and peak hand speed occurred earlier in downswing (evidenced by the increase in hand lag time). Although the changes in the magnitude and timing of the peak segmental velocities, in conjunction with the loss of range of torso and pelvis rotation, might have negative consequences on performance variables such as hitting distance, hitting distance was not specifically measured here. Future studies are warranted to examine the effects of putting practice on factors such as club head velocity and hitting distance.

Of the displacement variables examined, only upwards head movement and pelvis lateral flexion were significantly affected: the head did not move upwards as much postintervention and there was an increase in pelvis movement toward target (left) during the downswing. These findings are further evidence of an overall alteration in swing coordination, which suggests that these fatigueinduced compensatory strategies may affect performance.

Lindsay and Horton ${ }^{17}$ have reported a trend toward professional golfers with LBP having greater spinal flexion in the address position than those without LBP. In the present study, the majority of differences were observed during the downswing, although there were small changes in postural alignment in the address position. However, of the 8 variables examined, only pelvis flexion and head lateral flexion were significantly different postintervention. Although these changes were statistically significant, the practical significance of the pelvis being approximately $2^{\circ}$ less flexed and the head approximately $1^{\circ}$ less laterally flexed is unknown.

Previously, Horton et al ${ }^{9}$ compared the 


\section{[ RESEARCH REPORT ]}

effects of a practice session on abdominal muscle fatigue between golfers with chronic LBP and asymptomatic golfers. Following practice, which consisted of hitting golf balls with various clubs every 30 seconds for 50 minutes, neither group demonstrated significant abdominal muscle fatigue, but participants with chronic LBP reported a significant increase in the severity of their symptoms. The golfers in the present study were symptom free during testing, but research is needed to determine whether fatigue-related changes following putting practice increase the risk of experiencing LBP, or whether the changes are more pronounced in golfers with LBP. Also, given that golfers with high BMI were least affected by the intervention and have been found previously to develop less LBP, ${ }^{7}$ the nature of the relationship between the anthropometric characteristics of golfers, fatigue, and back pain warrants further examination.

The findings that a 40-minute putting task affected kinematics of full swings and resulted in significantly poorer performance on the Biering-Sørensen test support the idea that training programs for golfers that incorporate exercises intended to increase the fatigue threshold of the trunk extensors may reduce the effect of putting practice on swing performance. This hypothesis should be examined in future studies. Additionally, the findings of the present study have implications for future studies investigating optimal durations and structure of practice sessions where the goal is to prevent practice from affecting the quality of subsequent swing performance.

A limitation of the present study was that motion of the skin-mounted sensors relative to the underlying body segment may have resulted in errors in our kinematic data. These errors are difficult to avoid without using bone-mounted sensors but were minimized in the present study by mounting the sensors with a custom-made harness. The extent to which the anthropometric characteristics of individual participants influenced these skin motion errors was not assessed and there- fore a degree of bias in our kinematic data cannot be discounted. However, our reliability data and the consistent direction of our main findings give us confidence that skin motion artifact did not unduly influence the main conclusions of the study. A further limitation was that the single sensor attached to the torso at T3 might not have adequately reflected the true motion of the entire torso segment. Finally, projection angles, which were used to quantify 3-D motion of the torso, head, and trunk during each swing, can result in errors when rotations about the axis of interest are accompanied by large rotations about alternate 3-D axes. This issue is of less concern with the use of a repeatedmeasures (within-subjects) design, as was employed in the present study. However, care should be taken when comparing the angles reported here with those obtained using alternative methods.

\section{CONCLUSION}

N THIS STUDY, WE SHOWED THAT 40 minutes of putting significantly affected the kinematics of full golf swings made by skilled golfers and resulted in poorer performance on the Biering-Sørensen test. Immediately following the putting practice, movement coordination was altered, peak segmental speeds decreased, and swing duration increased. Future studies investigating the effects of these changes on club head velocity and hitting distance and accuracy are warranted. The findings here also show that a golfer's BMI influenced the changes observed in swing kinematics. That is, following putting practice, changes in torso and pelvis rotation range were smallest for golfers with high BMI. 0

\section{KEY POINTS}

FINDINGS: After 40 minutes of putting practice, the kinematics of 5-iron swings of skilled golfers were affected. The effects included reductions in the speed and magnitude of pelvis and torso axial rotation during the downswing. Golfers with higher BMI were least affected by putting practice.

IMPLICATION: The changes observed may have resulted from fatigue of the trunk extensors. Trunk muscle endurance training could help golfers maintain their swing kinematics after extended putting. Taller, thinner golfers may benefit most from such training.

CAUTION: Future studies should examine the effect putting practice has on club head speed and shot accuracy.

ACKNOWLEDGEMENTS: The authors acknowledge the National Health and Medical Research Council of Australia for their support of this project. The authors also wish to thank both Mr Ryan Lumsden (Golf BioDynamics Pty Ltd) and the Australian Professional Golfer's Association of Australia for their assistance.

\section{REFERENCES}

1. Bechler JR, Jobe FW, Pink M, Perry J, Ruwe PA. Electromyographic analysis of the hip and knee during the golf swing. Clin J Sport Med. 1995;5:162-166.

2. Biering-Sorensen F. Physical measurements as risk indicators for low-back trouble over a oneyear period. Spine. 1984;9:106-119.

3. Burden AM, Grimshaw PN, Wallace ES. Hip and shoulder rotations during the golf swing of sub-10 handicap players. J Sports Sci. 1998;16:165-176.

4. Cheetham PJ, Martin PE, Mottram RE, St Laurent BF. The importance of stretching the " $X$ Factor" in the downswing of golf: "The X-Factor stretch". In: Thomas PR, eds. Optimising Performance in Golf. Brisbane, QLD: Academic Press; 2001:192-199.

5. Demoulin C, Vanderthommen M, Duysens C, Crielaard JM. Spinal muscle evaluation using the Sorensen test: a critical appraisal of the literature. Joint Bone Spine. 2006;73:43-50. http://dx.doi.org/10.1016/j.jbspin.2004.08.002

6. Derksen JC, van Riel MP, Snijders CJ. A new method for continuous recording of trunk postures while playing golf. J Appl Biomech. 1996;12:116-129.

7. Evans K, Refshauge KM, Adams R, Aliprandi L. Predictors of low back pain in young. elite golfers: a preliminary study. Phys Ther Sport. 2005;6:122-130.

8. Forestier N, Nougier $\mathrm{V}$. The effects of muscular fatigue on the coordination of a multijoint movement in human. Neurosci Lett. 1998;252:187-190.

9. Horton JF, Lindsay DM, Macintosh BR. Abdomi- 
nal muscle activation of elite male golfers with chronic low back pain. Med Sci Sports Exerc. 2001;33:1647-1654.

10. Hosea TM, Gatt CJ, Galli KM, Langrana NA, Zawadsky JP. Biomechanical Analysis of the Golfer's Back. Glasgow, UK: E \& FN Spon; 1990.

11. Hume PA, Keogh J, Reid D. The role of biomechanics in maximising distance and accuracy of golf shots. Sports Med. 2005;35:429-449.

12. Jobe FW, Pink MM. Shoulder pain in golf. Clin Sports Med. 1996;15:55-63.

13. Kankaanpää M, Laaksonen D, Taimela S, Kokko SM, Airaksinen 0, Hanninen 0. Age, sex, and body mass index as determinants of back and hip extensor fatigue in the isometric Sфrensen back endurance test. Arch Phys Med Rehabil. 1998;79:1069-1075.

14. Keyserling WM, Sudarsan SP, Martin BJ, Haig AJ, Armstrong TJ. Effects of low back disability status on lower back discomfort during sustained and cyclical trunk flexion. Ergonomics. 2005;48:219-233. http://dx.doi. org/10.1080/0014013042000327689

15. Koumantakis GA, Arnall F, Cooper RG, Oldham JA. Paraspinal muscle EMG fatigue testing with two methods in healthy volunteers. Reliability in the context of clinical applications. Clin Biomech (Bristol, Avon). 2001;16:263-266.

16. Latimer J, Maher CG, Refshauge K, Colaco I. The reliability and validity of the Biering-Sørensen test in asymptomatic subjects and subjects reporting current or previous nonspecific low back pain. Spine. 1999;24:2085-2089; discussion 2090.

17. Lindsay D, Horton J. Comparison of spine motion in elite golfers with and without low back pain. J Sports Sci. 2002;20:599-605.

18. McHardy A, Pollard H. Muscle activity during the golf swing. Br J Sports Med. 2005;39:799-804; discussion 799-804. http://dx.doi.org/10.1136/ bjsm.2005.020271

19. McTeigue M, Lamb SR, Mottram RE, Pirozzolo F. Spine and hip motion analysis during the golf swing. In: Cochran AJ, Farrally MR, eds. Science and Golf II: Proceedings of the World Scientific Congress of Golf. London, UK: E \& FN Spon; 1994.

20. Moffroid M, Reid S, Henry SM, Haugh LD, Ricamato $A$. Some endurance measures in persons with chronic low back pain. J Orthop Sports Phys Ther. 1994;20:81-87.

21. Neal RJ, Wilson BD. 3D kinematics and kinetics of the golf swing. Int J Sport Biomech. 1985;1:221-232.

22. Pink M, Perry J, Jobe FW. Electromyographic analysis of the trunk in golfers. Am J Sports Med. 1993;21:385-388.

23. Provins KA. The specificity of motor skill and manual asymmetry: a review of the evidence and its implications. J Mot Behav. 1997;29:183-192.

24. Robinson RL. A study of the correlation between swing characteristics and club head velocity. In: Cochran AJ, Farrally MR, eds. Science and Golf
II: Proceedings of the World Scientific Congress of Golf. London, UK: E \& FN Spon; 1994.

25. Simmonds MJ, Olson SL, Jones S, et al. Psychometric characteristics and clinical usefulness of physical performance tests in patients with low back pain. Spine. 1998;23:2412-2421.

26. Slater-Hammel AT. Action current study of contraction-movement relationships in golf stroke. Res Q. 1948;19:166-177.

27. Sprigings EJ, Neal RJ. An insight into the importance of wrist torque in driving the golfball: a simulation study. J Appl Biomech. 2000;16:356-366.

28. Takahashi I, Kikuchi S, Sato K, Sato N. Mechanical load of the lumbar spine during forward bending motion of the trunk-a biomechanical study. Spine. 2006;31:18-23.

29. Theriault $\mathrm{G}$, Lachance P. Golf injuries. An overview. Sports Med. 1998;26:43-57.

30. van Ingen Schenau GJ, Bobbert MF, de Haan A. Does elastic energy enhance work and efficiency in the stretch-shorten cycle? J Appl Biomech. 1997;13:389-415.

31. Watkins RG, Uppal GS, Perry J, Pink M, Dinsay JM. Dynamic electromyographic analysis of trunk musculature in professional golfers. Am J Sports Med. 1996;24:535-538. 